\title{
Reduced-Order Modeling of Hypersonic Vehicle Unsteady Aerodynamics
}

\author{
Torstens Skujins * \\ Carlos E. S. Cesnik ${ }^{\dagger}$ \\ University of Michigan, Ann Arbor, Michigan 48109
}

\begin{abstract}
Accurate and computationally efficient models of unsteady aerodynamic loads are necessary for the development of hypersonic vehicle control algorithms. This work focuses on using convolution of modal step responses to construct a reduced-order model for these loads. In order to allow the model to be valid over a wide range of modal input amplitudes and flight conditions, a nonlinear correction factor is introduced. Not limited to a specific geometry, the correction factor methodology is general enough to be applied to many different two and three-dimensional vehicle configurations. Good correlation is seen between results obtained from the reduced-order model and computational results.
\end{abstract}

\section{Introduction}

The accurate prediction of aerodynamic loads is important for hypersonic vehicle control design and evaluation. Inaccurate representation of those loads could lead to inaccurate control algorithms and potentially loss of control of the vehicle. Hypersonic vehicles are flexible structures. Thus, in order to most accurately predict the aerodynamic loads, the dynamic deformations of the vehicle must be taken into consideration. Hypersonic vehicles are also highly coupled systems. Because of this, the inaccurate prediction of aerodynamic loads will have an adverse effect on the modeling of the structural dynamics, propulsion, thermodynamics, and other vehicle components, which would further inhibit the development of accurate control algorithms.

The calculation of unsteady aerodynamic loads has been approached in a number of different ways. The most computationally expensive method is to simply conduct full unsteady computational fluid dynamics (CFD) simulations. The major drawback of this method is that the time requirement can be prohibitively high for many cases, such as ones where several different model configurations need to be tested. Moreover, coupling with other disciplines is very challenging, if not unattainable. At the other end of the spectrum, simplified models such as piston theory ${ }^{1}$ provide quick, computationally efficient approximations with the tradeoff of a significant decrease in accuracy. It is also not feasible to quantify the error incurred using these simplified methods, as no reference case exists to compare the model against. In the middle are CFDbased reduced-order models (ROM). The goal of a CFD-based reduced-order model is to find the unsteady aerodynamic loads with accuracy approaching that of the CFD models but in a small fraction of the time. Unlike simplified models, a high-fidelity CFD reference case is constructed from which the error incurred by the ROM can be quantified.

Several different methods of reduced-order aerodynamic modeling have been developed. Proper orthogonal decomposition (POD) ${ }^{2,3,4}$ uses a series of flowfield snapshots to find the fluid basis vectors and hence significantly reduce the number of degrees of freedom in the system. Autoregressive moving average (ARMA) models find the response of a system based on a series of scaled system inputs and outputs. ${ }^{5,6}$

Another popular method has been the use of convolution and Volterra series. Silva ${ }^{7}$ developed a method to identify the Volterra series kernels based on the unit impulse or unit step responses obtained from CFD simulations. Then, linearized state-space models of the system were created first through mode-by-mode

\footnotetext{
*Graduate Research Assistant, University of Michigan, Email: tskujins@umich.edu, Member AIAA.

†Professor, Department of Aerospace Engineering, University of Michigan, Email: cesnik@umich.edu, 1320 Beal Avenue, 3024 FXB, Ann Arbor, MI 48109-2140, Ph. (734) 764-3397, Fax: (734) 764-0578, AIAA Associate Fellow.
} 
excitation of the system (one modal excitation per CFD run) ${ }^{8}$ and then by multiple modal inputs in a single CFD run, as developed by $\mathrm{Kim}^{9}$ and Silva. ${ }^{10}$ Munteanu et al. have also developed a Volterra-based kernel identification technique ${ }^{11}$ and state-space ROM. ${ }^{12}$ Lucia and Beran ${ }^{13}$ developed a method combining Volterra series with POD in order to more efficiently calculate a ROM in situations where response values need to be calculated in a large amount of locations, as in a large domain. These efforts were conducted primarily at the transonic regime and not at the hypersonic regime. This research focuses on the development of a convolution-type of ROM method for hypersonic unsteady aerodynamics.

A major drawback of reduced-order models is that they may only be valid for the specific Mach number, velocity, dynamic pressure, and other flight conditions at which the runs for model construction were conducted. This issue has been addressed in several different ways. Lind et al. ${ }^{14}$ use velocity curve fits of kernels identified from flight test data at different conditions to create velocity-independent kernels. Later, Baldelli et al. ${ }^{15}$ use a linear time invariant operator combined with a nonlinear operator to construct a model based on flight test data that is valid over a range of dynamic pressures. Prazenica et al. ${ }^{16}$ identify kernels from flight test data over a range of flight conditions and use curve fits to extrapolate the kernels to other flight conditions. Chung et al. ${ }^{17}$ include Mach number and altitude uncertainties in the $\mu$ method for finding flutter boundaries. Silva ${ }^{18}$ uses a single unsteady ROM over a range of velocities by simply modifying the sample rate within the state-space system due to the fact that the effect of velocity serves to modify the time step of numerical integration.

Another issue faced by convolution-type ROMs is that of amplitude of motion. If a modal step input of $a$ is used to construct a ROM for a nonlinear system, will the ROM still be valid for a modal input amplitude of, for example, 10a? The step input amplitudes for CFD runs are constrained in size by code limitations; too large of an input will result in the run blowing up. Silva recommends that the input amplitudes should be made as large as possible given code limitations and physical considerations. ${ }^{7}$ Raveh $^{19}$ states that, as expected, the most accurate ROM prediction generally occurs when the step input amplitude is very close to the amplitude of the arbitrary input being modeled. However, a ROM valid only for amplitudes near to those around which the ROM is constructed will inherently be limited. It is desired that the ROM be valid for a range of amplitudes of motion in addition to a range of flight conditions.

This work presents a CFD-based reduced-order model framework to calculate the unsteady lift, drag, and moment coefficients on a hypersonic vehicle that is valid over a wide range of modal amplitudes and Mach numbers. Additionally, though here it is only applied to a single two-dimensional geometry, the method is general enough to be applied to other two and three-dimensional configurations. While not intended for the initial stages of vehicle development, when many different vehicle configurations are being considered, the purpose of the ROM is to evaluate in a higher-fidelity manner the performance of a select number of configurations which have been chosen based on low-fidelity, simplified aerodynamics and structural testing.

\section{Reduced-Order Modeling Development}

\section{A. Convolution}

The response of a linear system to an arbitrary input can be found if the response of the system to a unit step $(H(t))$ or unit impulse $(h(t))$ function is known. The response $y(t)$ due to an arbitrary input $f(t)$ is found through the use of convolution: $:^{7,20}$

$$
y(t)=f(0) H(t)+\int_{0}^{t} \frac{d f}{d t}(\tau) H(t-\tau) d \tau
$$

Since the unit impulse is the derivative of the unit step, integration by parts yields

$$
y(t)=f(t) H(0)+\int_{0}^{t} f(\tau) h(t-\tau) d \tau
$$

Equations 1 and 2 are the two forms of Duhamel's integral.

The reduced-order model presented here is based on the convolution described above. Volterra series is the nonlinear extension of convolution. However, for the cases tested here, the ROM using convolution and the correction factor described in Section II.B.1 showed improved agreement with direct CFD results when compared with Volterra results. 


\section{B. ROM Construction}

The first step in the ROM construction process is to find the response to a step input of each elastic mode that will be considered. The step is chosen over the impulse because of difficulties obtaining a quality solution from the impulse input; this finding was also noted by Raveh. ${ }^{19}$ In order to construct the ROM, both steady and unsteady CFD runs are conducted. First, a steady-state solution is found at a desired flight condition. Then, the steady-state solution is used as the beginning of the unsteady run. The three different responses tracked are the lift, drag, and pitching moment coefficients.

For each mode, it is necessary to find the responses for both a positive and negative step input. The reason for this is the fact that that response to the negative step is not equal to the opposite of the positive step response. The positive step here is defined as one in which the front of the airfoil is deformed in the positive (up) direction, and vice-versa for the negative step (see Fig. 1).

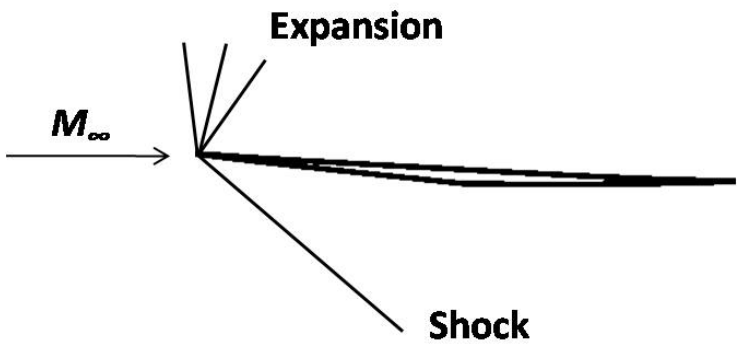

(a) Positive step

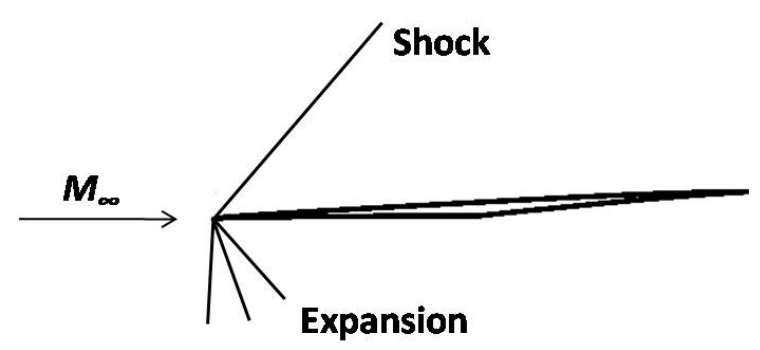

(b) Negative step

Figure 1. Modal step inputs

Once the step responses have been found, Eq. 1 is used to find the response to an arbitrary input. The response to an arbitrary elastic modal input is calculated two separate times, once using the positive step response in Eq. 1 and once using the negative step response. Suppose that the positive step causes an increase in some quantity $Q$ ( $Q$ may be $c_{l}, c_{d}$, etc.), and a negative step causes a decrease in the same quantity (from the value at the undeformed configuration). The positive response will be valid for situations when $Q$ is greater than the undeformed configuration value and vice-versa for the negative response.

Next, the ratio $r_{p n}$ between the maximum value of $Q$ found in the positive and negative convolved responses to the arbitrary input is calculated. The ROM can thus be calculated in two different ways. The first is to use the positive step response. Whenever $Q$ is above the undeformed value, the ROM will consist of the positive step response as is. However, when $Q$ drops below the undeformed value, the positive step response will be divided by $r_{p n}$. The second method is to use the negative step response when $Q$ is below the undeformed value and multiply by $r_{p n}$ when $Q$ is above it. When compared, the results of both methods are effectively equivalent.

\section{Correction Factor}

If left as described above, the ROM will only work for flight conditions and input amplitudes very near to those used for the step input. The responses do not scale linearly with amplitude. Figure 2 shows comparisons between the ROM coefficient responses with no nonlinear correction factor and direct CFD results at Mach 8 for the two-dimensional half-diamond airfoil geometry described in Section III.A.1 for two separate cases with sinusoidal modal inputs: one with maximum amplitude equal to the step input amplitude (Fig. 2(a)) and one with the maximum amplitude equal to $40 \times$ the step amplitude (Figs. 2(b) and 2(c)). The uncorrected ROM matches well to the CFD results in Fig. 2(a), as the lines are virtually on top of each other, but for the larger amplitude case of Fig. 2(b), the error becomes more significant. The drag coefficient response is not even close to the actual CFD results.

To account for this error, as well as error introduced by different flight conditions, a correction factor is introduced. Consider two step inputs of amplitudes $a_{1}$ and $a_{2}$. Step $a_{1}$ has the same amplitude and is at the flight condition as the one used to construct the ROM, while step $a_{2}$ can be at any flight condition within the flight envelope. Once the steps are applied, the responses will reach final, quasi-steady values $Y_{1}$ and $Y_{2}$, respectively. Before using these values for the correction factor, it is necessary to subtract off the initial 


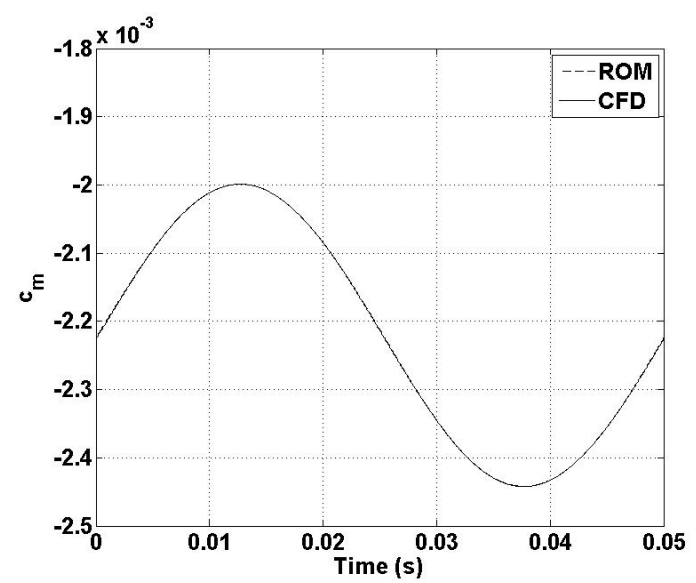

(a) $1 \times$ step amplitude

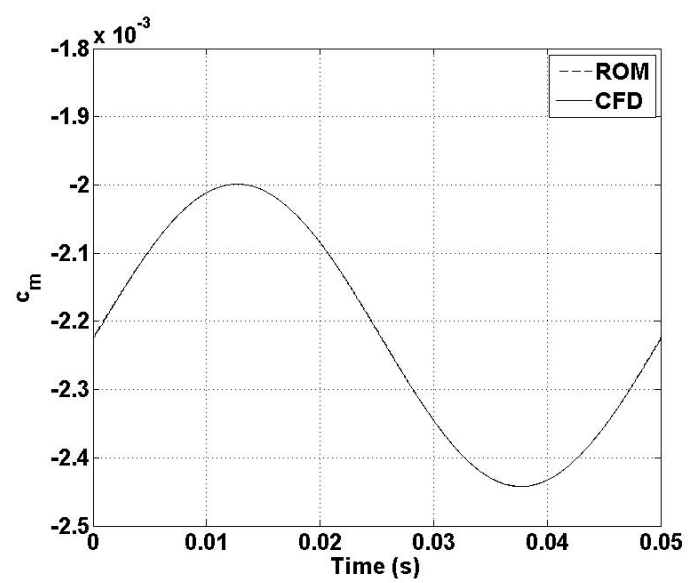

(b) $40 \times$ step amplitude, $c_{m}$

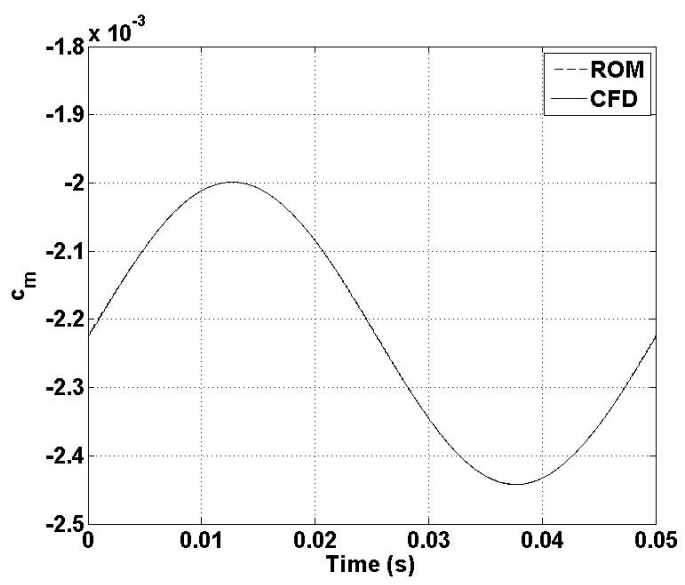

(c) $40 \times$ step amplitude, $c_{d}$

Figure 2. Uncorrected ROM responses 
steady-state values. These steady-state values function as a static offset in the convolution framework, so they must be subtracted off before convolution is performed and added back at the end of the process. Since the steady-state values of the coefficients will change with Mach number, it is thus necessary to conduct several steady runs and use polynomial curve fits in order to find the steady-state values throughout the range of interest. The values $y_{1}$ and $y_{2}$ used in the correction factor are $Y_{1}$ and $Y_{2}$ minus the steady-state values at $M_{1}$ and $M_{2}$, respectively. Figure 3 shows the curve fit, as well as the actual data points, for the moment coefficient.

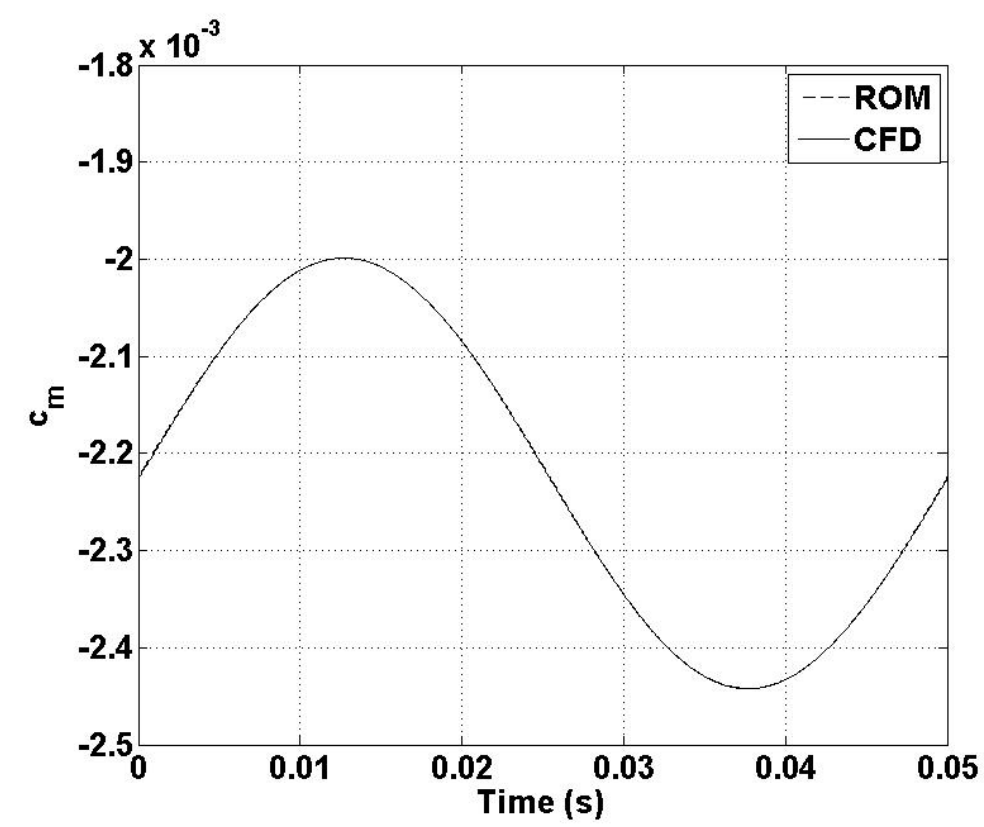

Figure 3. Steady-state values

The ROM correction factor $f_{c}$ is a ratio showing the scaling relationship between the input and output and is defined as follows:

$$
f_{c}=\frac{\frac{y_{2}}{y_{1}}}{\frac{a_{2}}{a_{1}}}
$$

Note that, for a truly linear system, $f_{c}=1$ (the response scales with the input). One issue in calculating the correction factor for certain amplitudes and flight conditions is that, if the amplitude is large enough, the CFD code will blow up when attempting to find the step response. However, the only value of interest for Eq. 3 is the final quasi-steady value of the response quantity. For this reason, it does not matter whether the modal deformation was a step or any other input. Because of this, values of $y_{2}$ in this study are found by sinusoidally increasing the modal input amplitude up to the desired maximum value. Once the maximum value is reached, the motion is stopped, and the quasi-steady response value is taken. Figure 4 shows a sample response used to find $y_{2}$ for an amplitude for which the step response would have blown up.

\section{Response Surface Construction}

In order to find the values of $f_{c}$ throughout the range of interest, a response surface is created with input amplitude and Mach number as the variables. Since $a_{1}$ (and hence $y_{1}$ ) are fixed at a reference amplitude and Mach number combination, the response surface is constructed by varying the parameters for $a_{2}$ throughout the flight envelope. However, when deciding upon the test points, it is important to ensure that the points are evenly distributed throughout the parameter space so that certain features of the response surface are not missed due to lack of testing in that specific area. To achieve this even spacing, nearly-orthogonal Latin hypercubes were used to determine the specific testing points; Ref. 21 provides a detailed discussion on their 


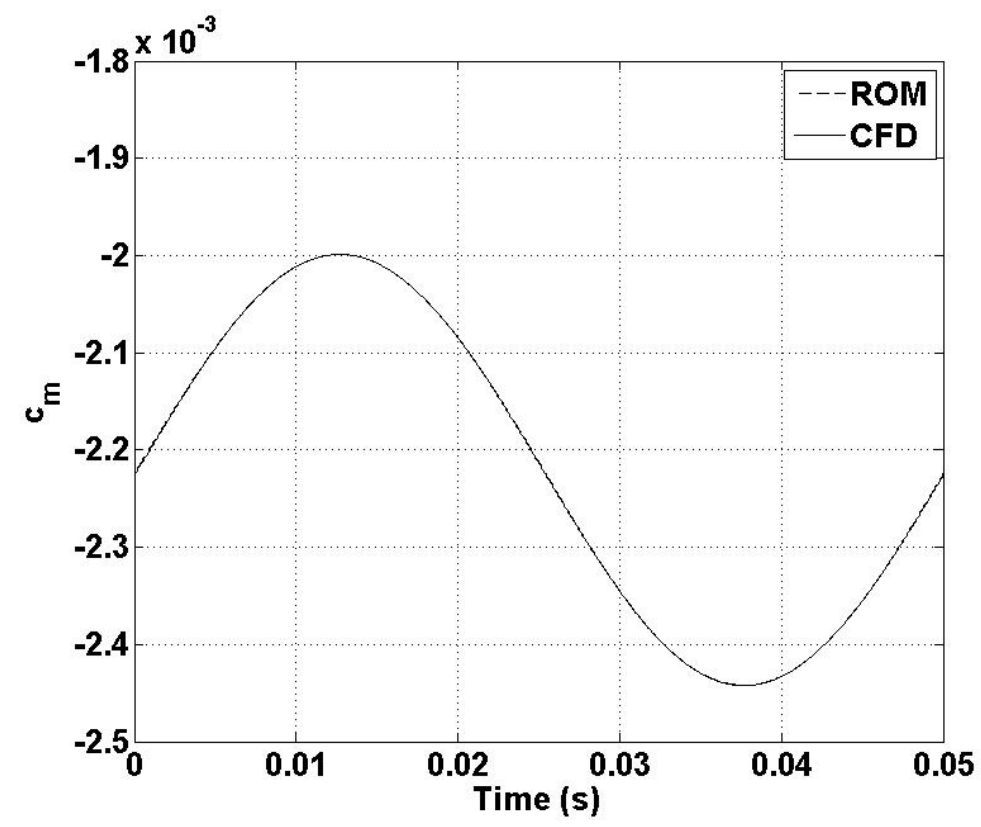

Figure 4. CFD response for finding $y_{2}, M=6.6$, amplitude $=100 \times$ step amplitude

design and construction. A spreadsheet created by Sanchez ${ }^{\mathrm{a}}$, which outputs the optimally-spaced testing points given a range of input parameters, is used for this purpose.

After the completion of the trials, the response surface is constructed using kriging. Unlike actual experiments, which inherently contain random error, computational simulations will give the same answer for the same simulation when repeated. Thus, a kriging response surface will include all test points on the surface, whereas a least squares fit surface will not in general directly pass through each point. For detailed explanations of kriging, see Refs. 22 and 23.

\section{Correction Factor Implementation}

After the response surface construction, the correction factor is implemented into the ROM. At each time step, the value of $f_{c}$ corresponding to the Mach number and input amplitude at that time step is obtained from the response surface. Rearranging Eq. 3, an expression for the corrected ROM value $y_{2}$ is obtained:

$$
y_{2}=f_{c} y_{1} \frac{a_{2}}{a_{1}}
$$

In this equation, $y_{1}$ is the step response value, while $\frac{a_{2}}{a_{1}}$ is the ratio of the current input amplitude to the step input amplitude. Thus, the quantity $y_{1} \frac{a_{2}}{a_{1}}$ is the linear, uncorrected ROM. To find $y_{2}$, the previouslycalculated, uncorrected ROM value is then multiplied by $f_{c}$. This process is repeated for each time step throughout the run.

\section{Numerical Studies}

To exemplify the process described above, a two-dimensional problem was created and is presented next. Note that the method is general and can be applied to three-dimensional configurations in a similar manner.

\footnotetext{
aSanchez, S. M., "NOLH designs spreadsheet," 2005. Available online via http://diana.cs.nps.navy.mil/SeedLab, Last accessed 06/22/2010.
} 


\section{A. Basic Problem Definition and Setup}

The CFD code used in this study is CFL3Dv6, developed at NASA Langley. ${ }^{24}$ The code is capable of solving the Euler/Navier-Stokes equations for both steady and unsteady flows on two and three-dimensional structured grids and has mesh deformation capability. Grids are created using the mesh generator ICEM CFD from ANSYS. ${ }^{25}$ All solutions used in this study are Euler solutions. Modal inputs are given to the airfoil geometry, described below, by utilizing the code's mesh deformation capabilities.

\section{Geometry}

The geometry used in this research is a two-dimensional half-diamond airfoil with a flat top surface. It is $2.5 \%$ thick and has a length of $1.6 \mathrm{~m}$. This is not intended to be representative of any specific airfoil or vehicle, as the method is general and can be applied to different configurations. The grid, shown in Fig. 5 (zoomed in on the airfoil) is a $548 \times 674$ structured grid with points concentrated more closely near the airfoil surface. The first mode step response obtained is virtually indistinguishable to that from a more refined grid of $644 \times 866$ points.

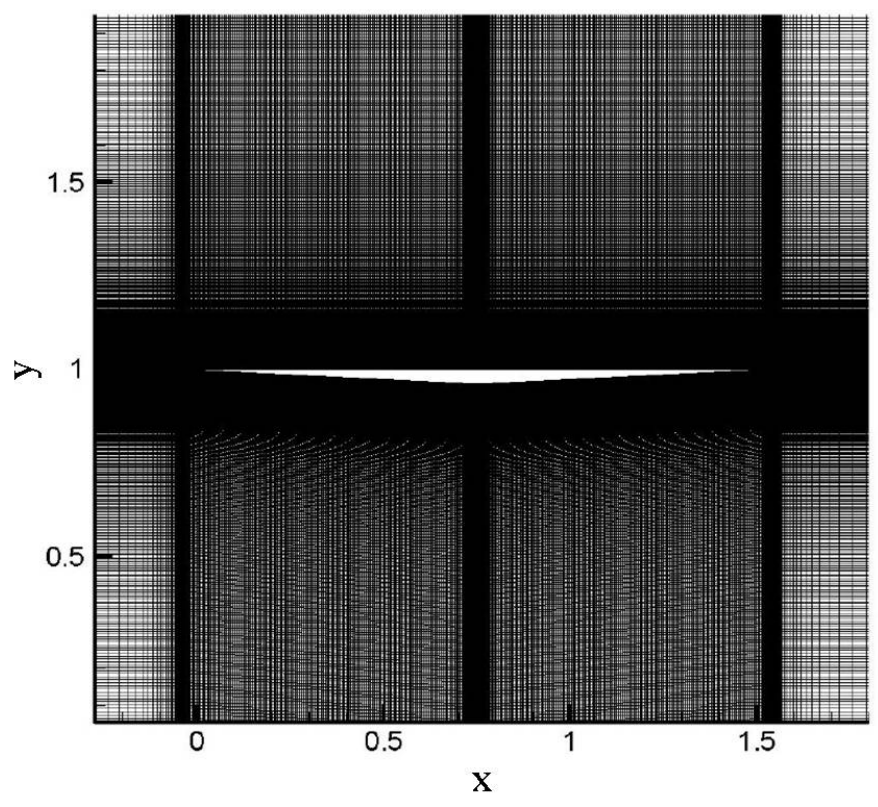

Figure 5. CFD half-diamond airfoil grid

\section{Mode Shapes}

Some fundamental deformation modes of the elastic structure must be used when creating the unsteady aerodynamic ROM. Typically, those fundamental modes are elastic mode shapes of the structure, and they would come from the solution of the structural dynamics part of the problem. To simulate those in our present study, two chordwise mode shapes were assumed. They were based on the first and third bending modes of a cantilever beam. Like the geometry itself, the mode shapes assumed here do not correspond to any specific configuration. Figure 6 shows a plot of the centerline displacements of these mode shapes; the amplitudes shown correspond to those used for the step inputs.

\section{B. Response Surface}

Response surfaces are constructed for both the first and third modes with $f_{c}$ corresponding to the lift, drag, and moment coefficients. For each case, two response surfaces are actually constructed, one including the positive amplitudes and one including the negative amplitudes. It was found that creating two surfaces 


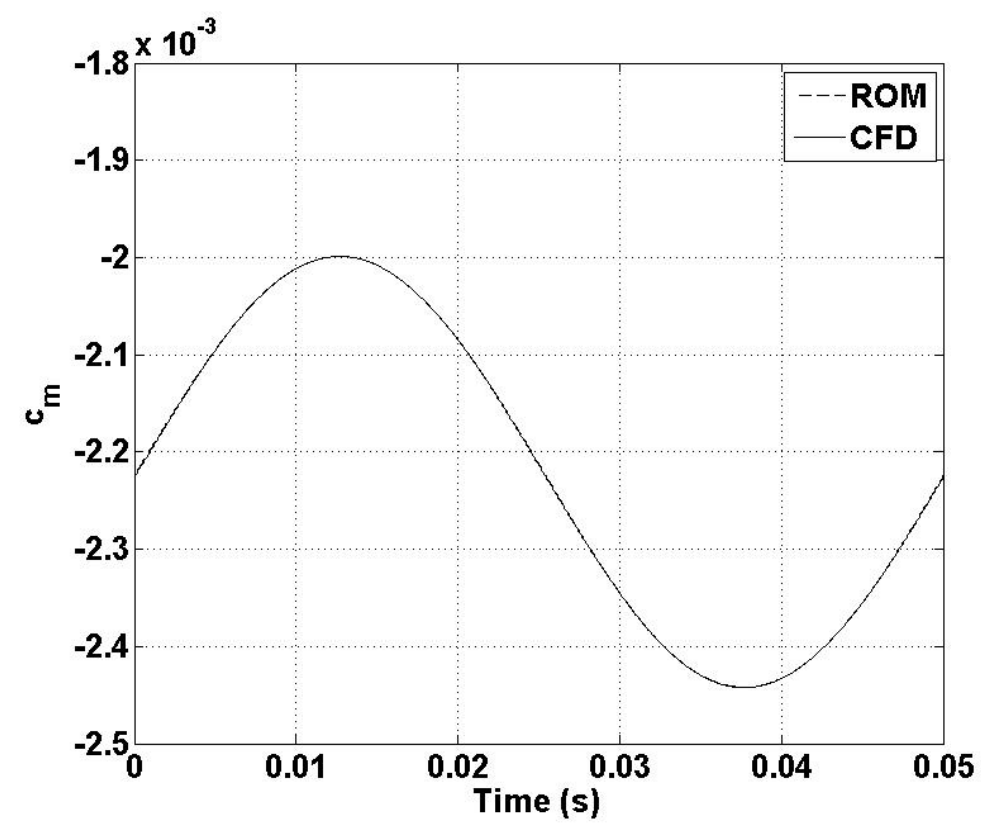

Figure 6. Mode shapes

resulted in each surface being smoother and thus producing more accurate results. The columns of Table 1 show the baseline (for $a_{1}$ and $y_{1}$ ) Mach number $M_{1}$, tip deflection corresponding to the step input $\left(a_{1}\right)$ in percent of chord length, range of Mach numbers, and amplitude range given in multiples of $a_{1}$. Note that all amplitudes in this section are given in multiples of the step amplitude. All runs have been conducted at zero angle of attack.

Table 1. Response surface parameters

\begin{tabular}{ccccc}
\hline \hline Mode & $M_{1}$ & $a_{1}$ & Mach range & Amplitude range \\
\hline 1 & 8 & $0.13 \% c$ & $5-9.5$ & $\pm 100 \times$ \\
3 & 8 & $0.08 \% c$ & $5-9.5$ & $\pm 40 \times$ \\
\hline \hline
\end{tabular}

Figure 7 shows the response surfaces for all three coefficients for the first mode. The dots are the actual ratio values calculated from CFD runs used to construct the surfaces. In addition to the Latin hypercube test point data, several further tests are conducted for $\pm 1 \times$ amplitude at various Mach numbers; since this is near the boundary between the positive and negative amplitude surfaces, and these regions of the response surfaces will be used often in ROM construction, additional points are used to improve accuracy in these areas.

\section{ROM vs. CFD Comparisons}

In order to test the accuracy of the ROM, the ROM results are compared with direct CFD simulations. As shown in Figs. 2(b) and 2(c), the main source of error when increasing the input amplitude using the uncorrected ROM is that ROM mispredicts the amplitude of the response. Thus, the error metric used to judge the accuracy of the corrected ROM will focus on this amplitude error. The error is defined by

$$
\text { error }=\max \left(\frac{\left|C F D_{\max }-R O M_{\max }\right|}{C F D_{\max }-C F D_{\min }}, \frac{\left|C F D_{\min }-R O M_{\min }\right|}{C F D_{\max }-C F D_{\min }}\right) \times 100 \%
$$




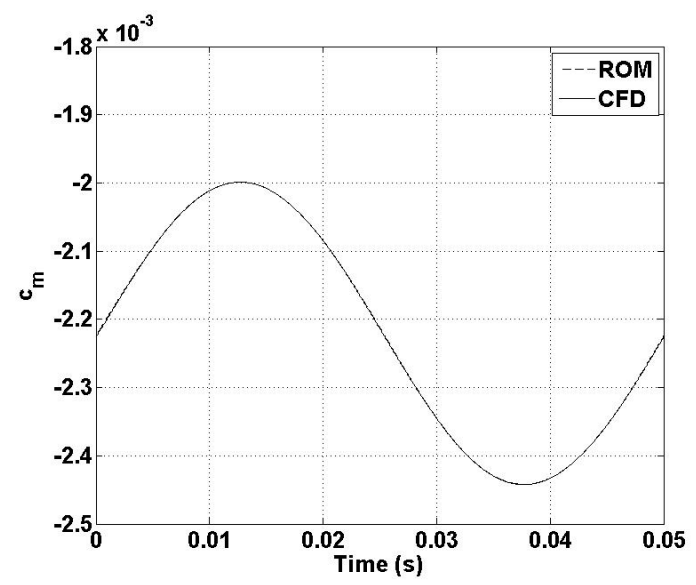

(a) $c_{l}$ response surface

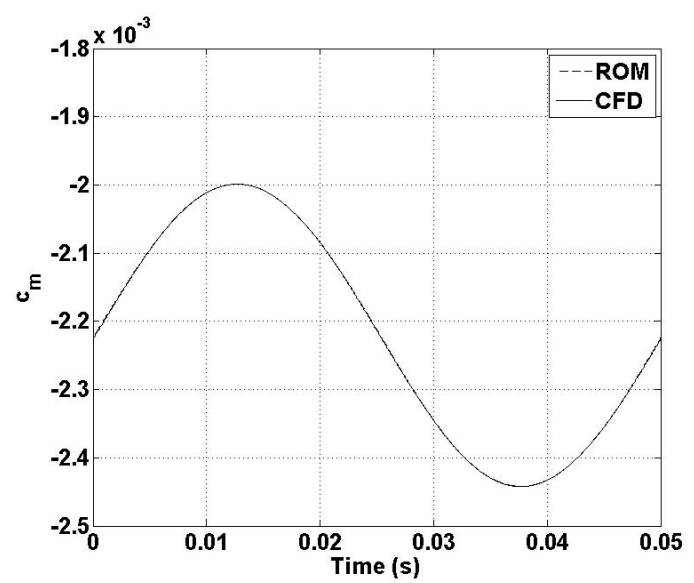

(b) $c_{d}$ response surface

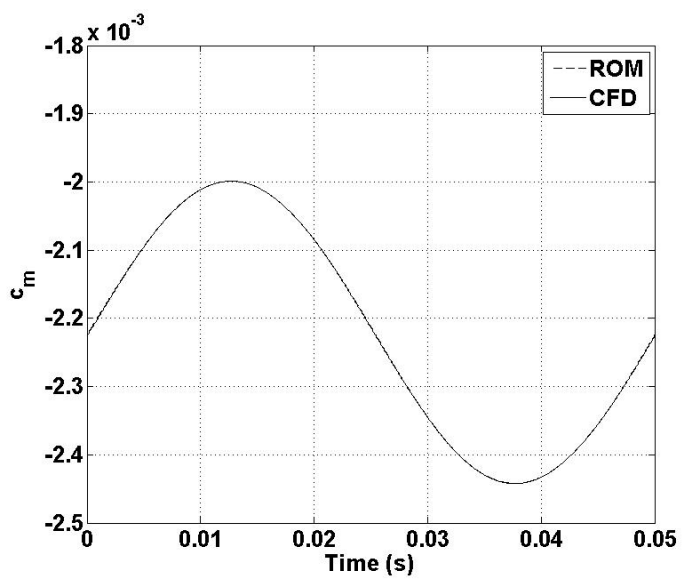

(c) $c_{m}$ response surface

Figure 7. Mode 1 response surfaces 
with the individual quantities within the equation defined in Fig. 8. Note that if other local peak values exist within the run (as in the drag coefficient cases), the error is calculated at those locations as well.

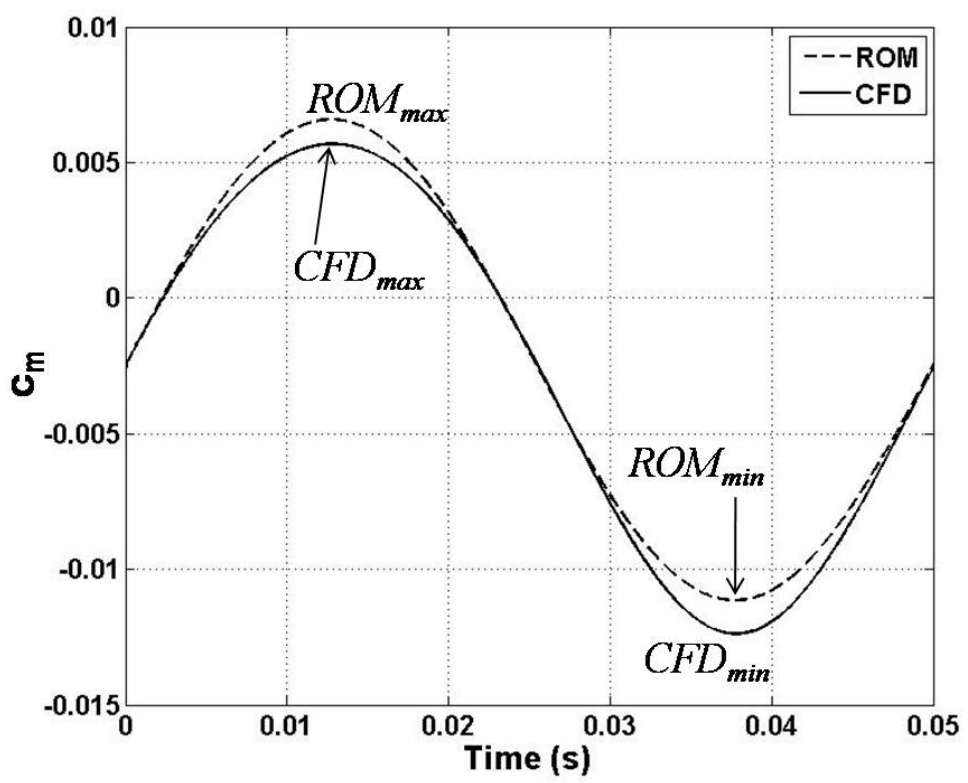

Figure 8. ROM-CFD comparison error metric

The first test of the correction factor is to see how it compares with the uncorrected ROM throughout the range of amplitudes for a given Mach number. Tests are conducted at Mach 8 of sinusoidal inputs with amplitudes varying from the step amplitude up to the maximum amplitudes used in the response surfaces for modes 1 and 3 (see Table 1). Figures 9 and 10 show the percent errors of each of the three coefficients for the first and third modes, respectively.

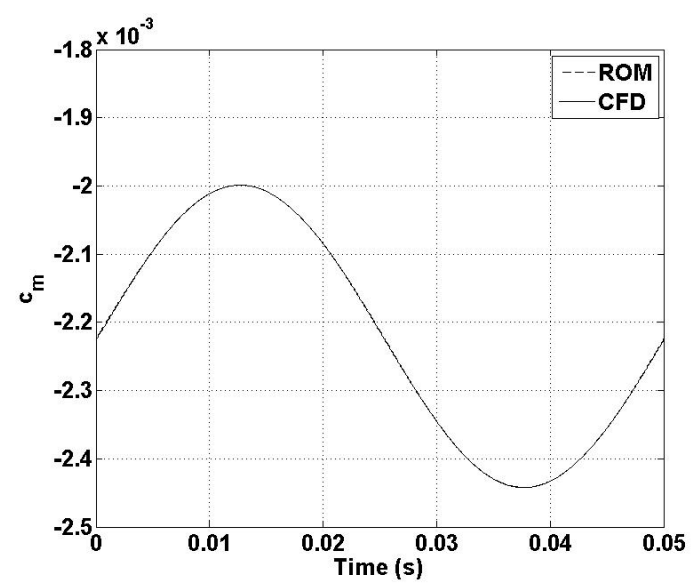

(a) $c_{l}$ and $c_{m}$ errors

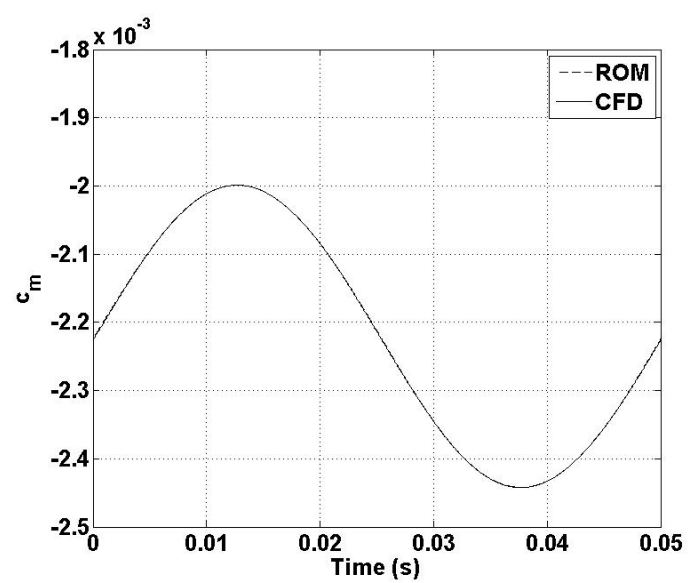

(b) $c_{d}$ error

Figure 9. Error between the ROM and direct CFD results for mode 1, $\omega=125.7 \mathrm{rad} / \mathrm{s}$

The improvement of the ROM by including the correction factor is drastic. The errors seen are generally lower than $1 \%$ for the corrected ROM, while the uncorrected ROM's errors continually increase. Figure 11 shows the ROM the the CFD simulations for a sinusoidal excitation of the first mode with an amplitude of $40 \times$ (the same case as plotted in Fig. 2). The uncorrected ROM showed significant amplitude errors, while 


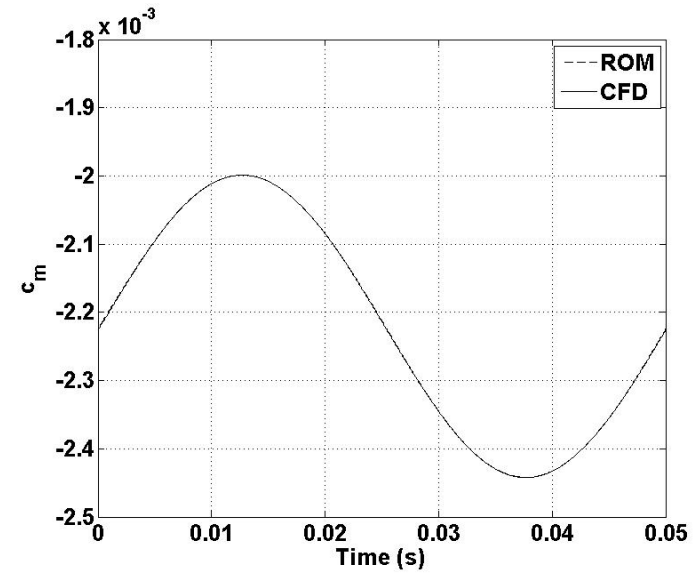

(a) $c_{l}$ and $c_{m}$ errors

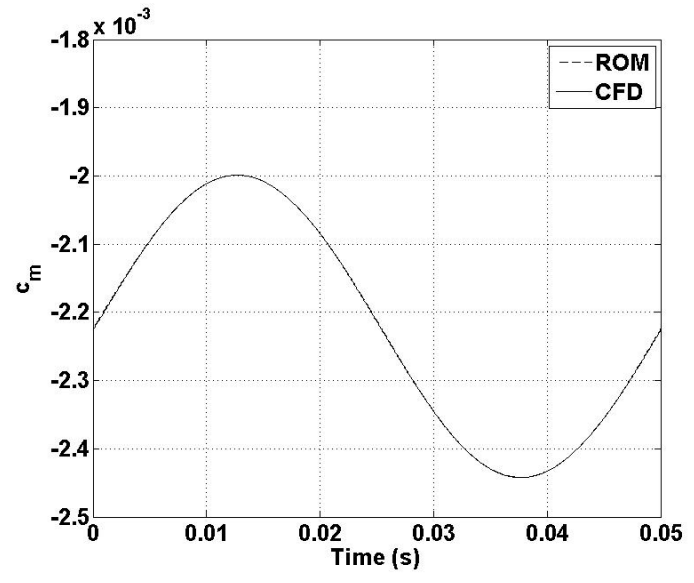

(b) $c_{d}$ error

Figure 10. Error between the ROM and direct CFD results for mode 3, $\omega=251.4 \mathrm{rad} / \mathrm{s}$

the corrected ROM lies virtually on top of the CFD results for each of the coefficients.

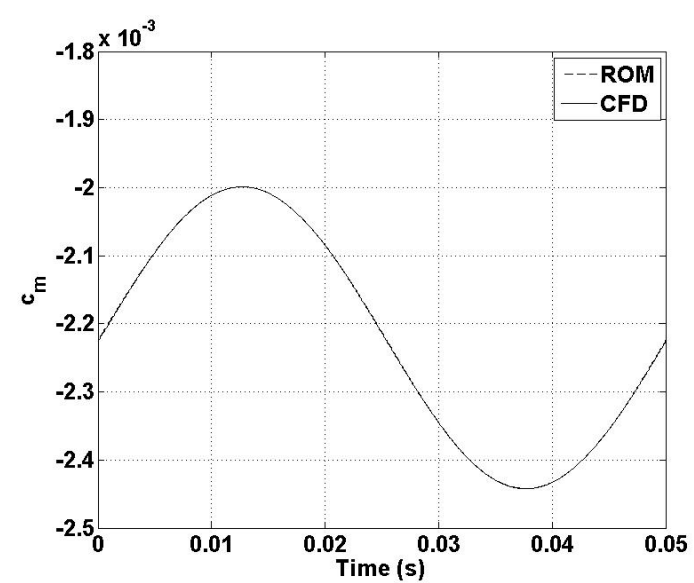

(a) $c_{m}$ comparison

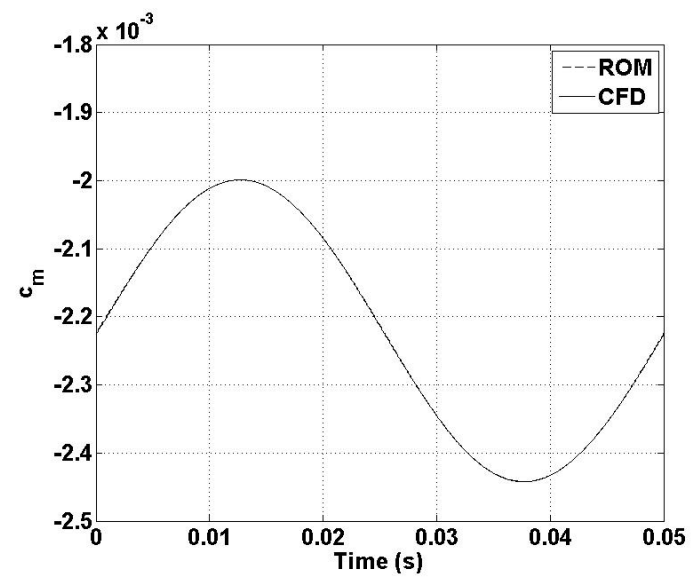

(b) $c_{d}$ comparison

Figure 11. Time domain results based on the ROM and direct CFD simulations for mode 1 , amplitude $=40 \times$, $M=8$

Test cases at other Mach numbers are conducted as well. Table 2 displays the parameters and errors. As in the cases above, good overall agreement exists between the ROM and CFD results. Figure 12(a) shows the unsteady drag obtained from the ROM and CFD solutions for case 1 from Table 2. One noticeable feature about the comparison is that, while ROM captures the amplitude very well, a slight phase shift exists between the two solutions. To investigate whether the frequency played a role in this shift, another test case with the same parameters as case 1 but with $\omega=125.7 \mathrm{rad} / \mathrm{s}$ is conducted. That comparison is shown in Fig. 12(b).

To quantify the error caused by the phase shift, a maximum percent error metric is introduced. This is similar to the metric described previously, but any time step is used rather than just the peaks. The maximum percent error falls from $4.20 \%$ for the higher frequency $(251.0 \mathrm{rad} / \mathrm{s})$ to $2.14 \%$ for the lower frequency $(125.7 \mathrm{rad} / \mathrm{s})$. The same finding holds for case 2 as well, as the error falls from $3.72 \%$ at $\omega=251.0 \mathrm{rad} / \mathrm{s}$ to $1.71 \%$ at $\omega=125.7 \mathrm{rad} / \mathrm{s}$. However, this error reduction also may be due to the fact that the responses for higher frequencies have higher gradients in time. The same value of shift will produce a higher percent 
Table 2. Test Cases, Mode 1, $\omega=251.0 \mathrm{rad} / \mathrm{s}$

\begin{tabular}{cccccc}
\hline \hline Case & $M$ & Amplitude & $c_{l}$ error (\%) & $c_{d}$ error (\%) & $c_{m}$ error (\%) \\
\hline 1 & 5 & $33 \times$ & 0.35 & 0.70 & 0.22 \\
2 & 6 & $55 \times$ & 0.37 & 0.36 & 0.39 \\
3 & 6.9 & $81 \times$ & 0.21 & 0.56 & 0.25 \\
4 & 7 & $72 \times$ & 0.22 & 0.22 & 0.20 \\
5 & 9 & $46 \times$ & 0.13 & 0.34 & 0.08 \\
\hline \hline
\end{tabular}

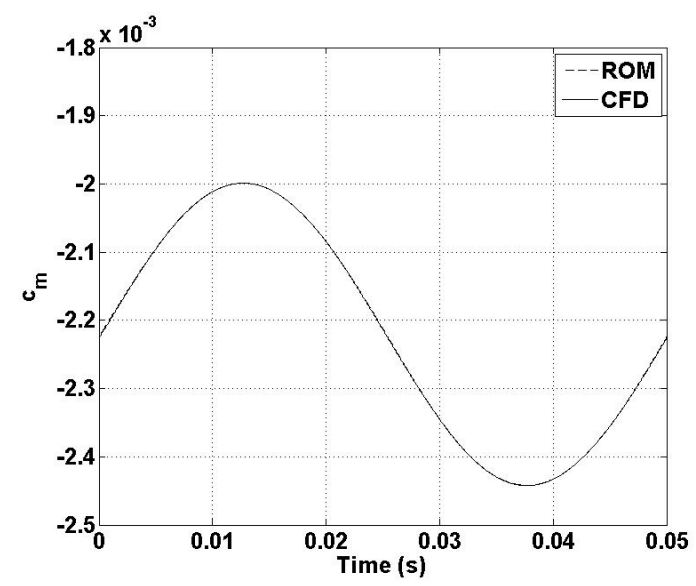

(a) $\omega=251.0 \mathrm{rad} / \mathrm{s}$

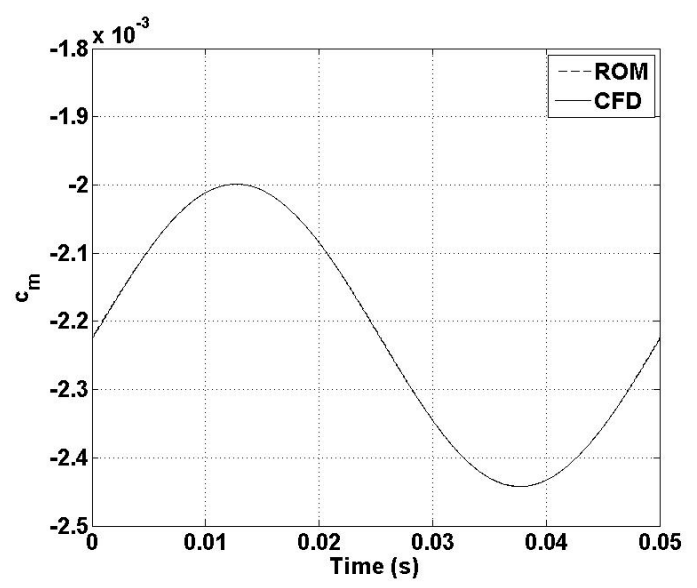

(b) $\omega=125.7 \mathrm{rad} / \mathrm{s}$

Figure 12. Frequency impact on the relative phase between ROM and CFD simulations for Case 1 ( $M=5$, amplitude $=33 \times)$ 
error for cases with higher slopes than those with lower slopes, as illustrated in Fig. 13. For the same shift value $\delta, a>b$.
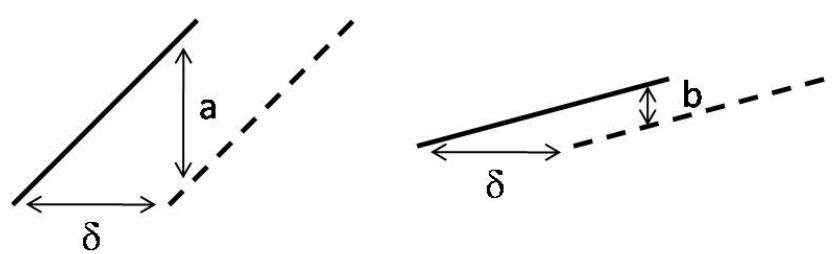

Figure 13. Shift error illustration

Table 3 shows the parameters and error results for cases with varying Mach number and amplitude for the third mode.

Table 3. Test Cases, Mode 3, $\omega=251.0 \mathrm{rad} / \mathrm{s}$

\begin{tabular}{cccccc}
\hline \hline Case & $M$ & Amplitude & $c_{l}$ error $(\%)$ & $c_{d}$ error (\%) & $c_{m}$ error (\%) \\
\hline 6 & 5 & $7 \times$ & 0.39 & 1.24 & 0.53 \\
7 & 6 & $22 \times$ & 0.23 & 0.20 & 2.62 \\
8 & 7 & $31 \times$ & 0.18 & 0.25 & 3.71 \\
9 & 9 & $12 \times$ & 0.28 & 0.54 & 0.22 \\
\hline \hline
\end{tabular}

Overall, the agreement is again very good. Figure 14 shows the ROM and CFD simulations for the drag and moment coefficients for case 6 . The moment coefficient plot does show a phase shift between the ROM and CFD results. However, for the drag coefficient, the shift does not appear. As in the cases for the first mode, cases 6 and 8 are re-conducted at a lower frequency $(125.7 \mathrm{rad} / \mathrm{s})$. The maximum $c_{m}$ percent error for case 6 falls from $5.18 \%$ at $\omega=251.0 \mathrm{rad} / \mathrm{s}$ to $3.09 \%$ at $\omega=125.7 \mathrm{rad} / \mathrm{s}$. Slight reductions seen in the other coefficients as well $\left(1.43 \%\right.$ to $0.83 \%$ for $c_{l}$ and $1.37 \%$ to $1.25 \%$ for $\left.c_{d}\right)$, but the errors for those are relatively small from the outset.

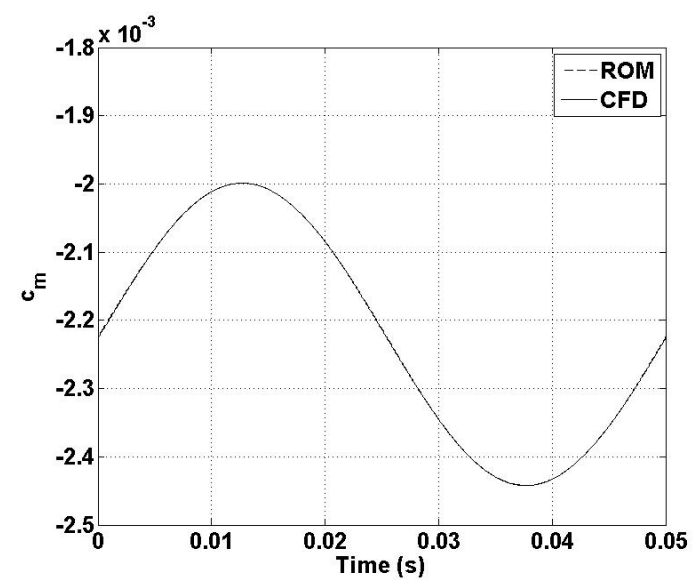

(a) $c_{d}$ comparison

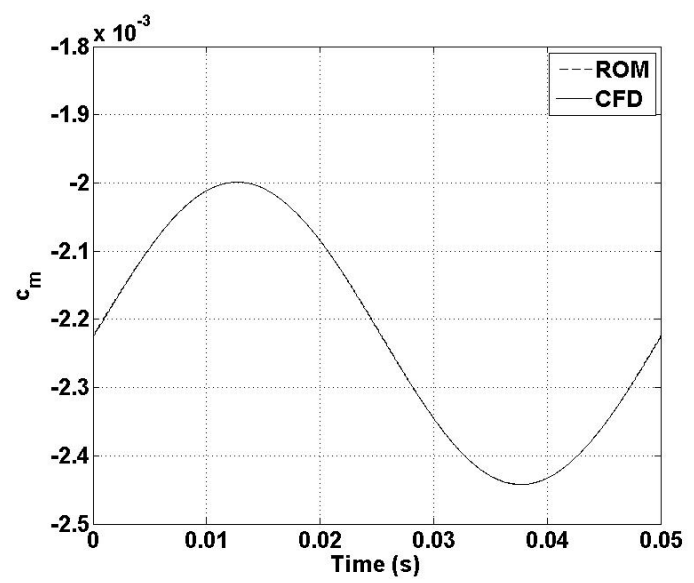

(b) $c_{m}$ comparison

Figure 14. Time domain results based on the ROM and direct CFD simulations for mode 3, amplitude $=7 \times$, $M=5$

The CFD results also show that, as the amplitude of motion increases, the shape of the moment coefficient response changes. Figure 15 shows the moment response from two test cases, both at Mach 8 and having 
a modal input frequency of $251.4 \mathrm{rad} / \mathrm{s}$; however, one has an input amplitude of $4 \times$, and the other has an amplitude of $40 \times$. Both of these trials are included in Fig. 10. The agreement is very good for the $4 \times$ case (Fig. 15(a)). For the $40 \times$ case, though, the shape of the response has changed somewhat, a phenomena not captured completely by the ROM. This behavior is not seen in the lift or drag coefficients or in the first mode moment coefficient.

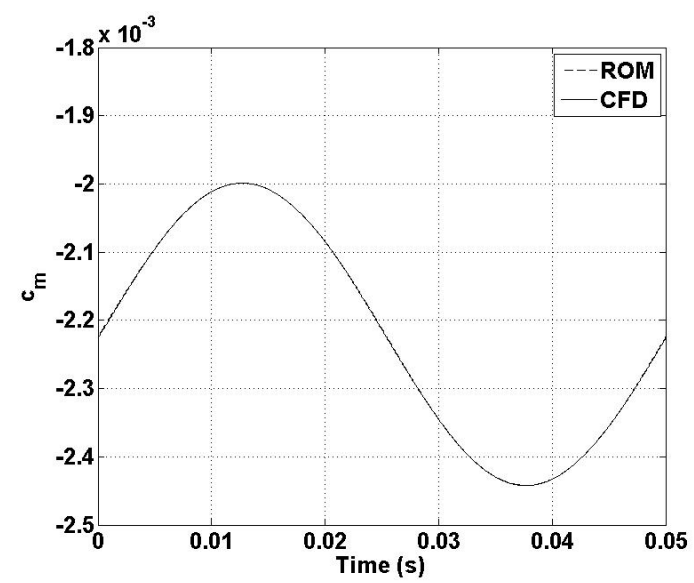

(a) Amplitude $4 \times$

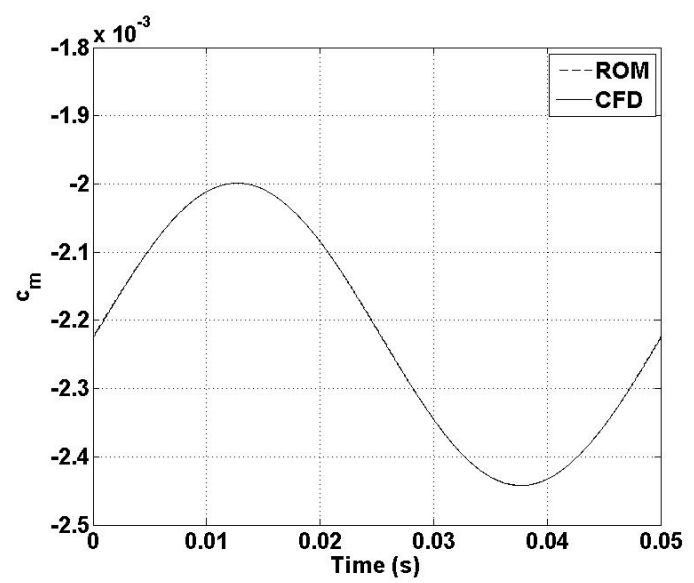

(b) Amplitude 40x

Figure 15. Amplitude impact on the moment coefficient $(M=8, \omega=251.4 \mathrm{rad} / \mathrm{s})$

It is also worthy to note that, though the response surface does not include altitude, these results are altitude-independent as well. The test cases shown have all been conducted at 85, $000 \mathrm{ft}$, but the coefficients do not change for runs with the same modal inputs and Mach numbers at different altitudes. However, for viscous solutions, viscosity, and thus the coefficients, will change with altitude. In those cases, altitude can be added in as an additional flight condition variable.

\section{Concluding Remarks}

A convolution-based reduced-order model for hypersonic vehicle unsteady aerodynamics has been presented. While here applied to a simple two-dimensional half-diamond airfoil configuration, the method is general enough to be applied to other two and three-dimensional configurations. Rather than being limited to flight conditions and amplitudes of motion close to those around which it was constructed, the ROM is valid for a wide range of flight conditions and input amplitudes. This is accomplished through the use of a nonlinear correction factor. The first two elastic modes of a two-dimensional half-diamond airfoil were considered. The important conclusions are as follows:

- Overall agreement between ROM and CFD for lift, drag, and moment coefficients for sinusoidal oscillation of each mode was very good. Errors throughout the amplitude and Mach number range tested typically were on the order of $1 \%$.

- The correction factor resulted in a significant improvement over uncorrected ROM, especially for larger input amplitudes.

- The accuracy of the ROM seems to have a slight frequency dependence, as the higher frequency runs have a slight phase shift that is not seen as much in the lower frequency runs. However, even for those cases, errors are still relatively small.

- For high input amplitudes of the third mode, the moment coefficient response changed shape, which wasn't captured by the ROM. The possibility of this happening will need to be monitored for future applications. 
Future work will consist of investigating multiple-modal oscillations, the inclusion of the full Navier-Stokes equations, and application to more complex two and three-dimensional geometries.

\section{Acknowledgments}

Funds for the Michigan-AFRL Collaborative Center in Control Science (MACCCS) were made available from the Air Force Research Laboratory/Air Vehicles Directorate grant number FA 8650-07-2-3744. The program manager is Michael Bolender.

\section{References}

${ }^{1}$ Ashley, H. and Zartarian, G., "Piston Theory - A New Aerodynamic Tool for the Aeroelastician," Journal of the Aeronautical Sciences, Vol. 23, No. 12, 1956, pp. 1109-1118.

${ }^{2}$ Lucia, D., Beran, P., and Silva, W., "Reduced-order modeling: new approaches for computational physics," Progress in Aerospace Sciences, Vol. 40, 2004, pp. 51-117.

${ }^{3}$ Thomas, J., Dowell, E., and Hall, K., "Three-Dimensional Transonic Aeroelasticity Using Proper Orthogonal Decomposition-Based Reduced-Order Models," Journal of Aircraft, Vol. 40, No. 3, May-June 2003, pp. 544-551.

${ }^{4}$ Farhat, C. and Amsallem, D., "Recent Advances in Reduced-Order Modeling and Application to Nonlinear Computational Aeroelasticity," Proceedings of the 46th AIAA Aerospace Sciences Meeting and Exhibit, AIAA Paper No. 2008-562, January 2008.

${ }^{5}$ Cowan, T., A.S. Arena, J., and Gupta, K., "Accelerating Computational Fluid Dynamics Based Aeroelastic Predictions Using System Identification," Journal of Aircraft, Vol. 38, No. 1, January-February 2001, pp. 81-87.

${ }^{6}$ Gupta, K. and Bach, C., "Systems Identification Approach for a Computational-Fluid-Dynamics-Based Aeroelastic Analysis," AIAA Journal, Vol. 45, No. 12, December 2007, pp. 2820-2827.

${ }^{7}$ Silva, W., "Discrete-Time Linear and Nonlinear Aerodynamic Impulse Responses for Efficient CFD Analyses," Ph.D. dissertation, College of William \& Mary, December 1997.

${ }^{8}$ Silva, W. and Bartels, R., "Development of reduced-order models for aeroelastic analysis and flutter prediction using the CFL3Dv6.0 code," Journal of Fluids and Structures, Vol. 19, 2004, pp. 729-745.

${ }^{9}$ Kim, T., "Efficient Reduced-Order System Identification for Linear Systems with Multiple Inputs," AIAA Journal, Vol. 43, No. 7, July 2005, pp. 1455-1464.

${ }^{10}$ Silva, W., "Simultaneous Excitation of Multiple-Input/Multiple-Output CFD-Based Unsteady Aerodynamic Systems," Journal of Aircraft, Vol. 45, No. 4, July-August 2008, pp. 1267-1274.

${ }^{11}$ Munteanu, S., Rajadas, J., Nam, C., and Chattopadhyay, A., "Reduced-Order-Model Approach for Aeroelastic Analysis Involving Aerodynamic and Structural Nonlinearities," AIAA Journal, Vol. 43, No. 3, March 2005, pp. 560-571.

${ }^{12}$ Munteanu, S., Rajadas, J., and Chattopadhyay, A., "Nonlinear Aeroelastic Analysis Using a CFD-Based State Space ROM," Proceedings of the 47th AIAA/ASME/ASCE/AHS/ASC Structures, Structural Dynamics, and Materials Conference, AIAA Paper No. 2006-2024, May 2006.

${ }^{13}$ Lucia, D. and Beran, P., "Reduced-Order Model Development Using Proper Orthogonal Decomposition and Volterra Theory," AIAA Journal, Vol. 42, No. 6, June 2004, pp. 1181-1190.

${ }^{14}$ Lind, R., Prazenica, R., Brenner, M., and Baldelli, D., "Identifying Parameter-Dependent Volterra Kernels to Predict Aeroelastic Instabilities," AIAA Journal, Vol. 43, No. 12, December 2005, pp. 2496-2502.

${ }^{15}$ Baldelli, D., Zeng, J., Lind, R., and Harris, C., "Flutter-Prediction Tool for Flight-Test-Based Aeroelastic ParameterVarying Models," Journal of Guidance, Control, and Dynamics, Vol. 32, No. 1, January-February 2009, pp. $158-171$.

${ }^{16}$ Prazenica, R., Reisenthel, P., Kurdila, A., and Brenner, M., "Volterra Kernel Extrapolation for Modeling Nonlinear Aeroelastic Systems at Novel Flight Conditions," Journal of Aircraft, Vol. 44, No. 1, January-February 2007, pp. 149-162.

${ }^{17}$ Chung, C., Shin, S., and Kim, T., "A New Robust Aeroelastic Analysis Including Aerodynamic Uncertainty from Varying Mach Numbers," Proceedings of the 49th AIAA/ASME/ASCE/AHS/ASC Structures, Structural Dynamics, and Materials Conference, AIAA Paper No. 2008-2200, April 2008.

${ }^{18}$ Silva, W., "Recent Enhancements to the Development of CFD-Based Aeroelastic Reduced-Order Models," Proceedings of the 48th AIAA/ASME/ASCE/AHS/ASC Structures, Structural Dynamics, and Materials Conference, AIAA Paper No. 2007-2051, April 2007.

${ }^{19}$ Raveh, D., "Reduced-Order Models for Nonlinear Unsteady Aerodynamics," AIAA Journal, Vol. 39, No. 8, August 2001, pp. $1417-1429$.

${ }^{20}$ Fung, Y., An Introduction to the Theory of Aeroelasticity, Dover Publications, Mineola, New York, 1993.

${ }^{21}$ Cioppa, T. and Lucas, T., "Efficient Nearly Orthogonal and Space-Filling Latin Hypercubes," Technometrics, Vol. 49, No. 1, February 2007, pp. 45-55.

${ }^{22}$ Lophaven, S., Nielsen, H., and Sondergaard, J., "DACE: A MATLAB Kriging Toolbox, Version 2.0," Tech. Rep. IMMTR-2002-12, Informatics and Mathematical Modelling, Denmark, August 1, 2002.

${ }^{23}$ Sacks, J., Welch, W., Mitchell, T., and Wynn, H., "Design and Analysis of Computer Experiments," Statistical Science, Vol. 4, No. 4, 1989, pp. 409-435.

${ }^{24}$ Rumsey, C. and Biedron, R., "CFL3D User's Manual, Version 5.0, Second Edition," Tech. Rep. Hampton, VA, NASA Langley Research Center, September 1997 (last updated August 2009).

${ }^{25}$ Anon., "Documentation for ANSYS ICEM CFD 12.0," Tech. rep., ANSYS, Canonsburg, Pennsylvania, 2009. 